\title{
Germline Proliferation Is Regulated by Somatic Endocytic Genes via JNK and BMP Signaling in Drosophila
}

\author{
Yaning Tang, ${ }^{*,+1}$ Qing Geng, ${ }^{*, 1,2}$ Di Chen, ${ }^{+}$Shaowei Zhao, ${ }^{+}$Xian Liu, ${ }^{\dagger}$ and Zhaohui Wang ${ }^{* 2}$ \\ *State Key Laboratory of Molecular Developmental Biology, Institute of Genetics and Developmental Biology, Chinese Academy of \\ Sciences, Chaoyang, Beijing, China 100101 and †University of Chinese Academy of Sciences, Huairou, Beijing, China 101408
}

\begin{abstract}
Signals derived from the microenvironment contribute greatly to tumorigenesis. The underlying mechanism requires thorough investigation. Here, we use Drosophila testis as a model system to address this question, taking the advantage of the ease to distinguish germline and somatic cells and to track the cell numbers. In an EMS mutagenesis screen, we identified Rab5, a key factor in endocytosis, for its nonautonomous role in germline proliferation. The disruption of Rab5 in somatic cyst cells, which escort the development of germline lineage, induced the overproliferation of underdifferentiated but genetically wild-type germ cells. We demonstrated that this nonautonomous effect was mediated by the transcriptional activation of Dpp [the fly homolog of bone morphogenetic protein (BMP)] by examining the Dpp-reporter expression and knocking down Dpp to block germline overgrowth. Consistently, the protein levels of Bam, the germline prodifferentiation factor normally accumulated in the absence of BMP/Dpp signaling, decreased in the overproliferating germ cells. Further, we discovered that the JNK signaling pathway operated between Rab5 and Dpp, because simultaneously inhibiting the JNK pathway and Rab5 in cyst cells prevented both dpp transcription and germline tumor growth. Additionally, we found that multiple endocytic genes, such as avl, TSG 101, Vps25, or Cdc42, were required in the somatic cyst cells to restrict germline amplification. These findings indicate that when the endocytic state of the surrounding cells is impaired, genetically wild-type germ cells overgrow. This nonautonomous model of tumorigenesis provides a simple system to dissect the relation between tumor and its niche.
\end{abstract}

KEYWORDS Rab5; BMP/Dpp; JNK pathway; tumorigenesis; nonautonomous

ICROENVIRONMENT exerts a great influence on tumor growth. The cancer microenvironment, also known as the cancer stem cell niche, includes the extracellular matrix, vasculature, immune system, etc. (Reilly and Van Dyke 2008). The genetic state of cells in the microenvironment could favor or impede tumorigenesis (Tabassum and Polyak 2015; Nemeth et al. 2016; Tissot et al. 2016). Thus, the mutual interactions between tumor and the cellular environment contribute greatly in tumor initiation, progression, and metastasis. A thorough understanding of how the nonautonomous effect

Copyright @ 2017 by the Genetics Society of America

doi: https://doi.org/10.1534/genetics.116.196535

Manuscript received October 5, 2016; accepted for publication March 6, 2017; published Early Online March 17, 2017.

Available freely online through the author-supported open access option

Supplemental material is available online at www.genetics.org/lookup/suppl/doi:10.

1534/genetics.116.196535/-/DC1.

${ }^{1}$ These authors contributed equally to this work.

${ }^{2}$ Corresponding authors: Chinese Academy of Sciences, BeiChenXiLu\#1, Beijing

100101, PR China. E-mail: zhwang@genetics.ac.cn; and qgeng@genetics.ac.cn coming from microenvironment promotes tumorigenesis may provide strategies for tumor treatment.

Drosophila is an excellent system to study tumorigenesis, because many human oncogenic genes and tumor suppressor genes are conserved (Banfi et al. 1996; Rubin et al. 2000; Ito et al. 2014). The fly testis is a regenerative tissue. Germ cells differentiate in an intimate contact with somatic cells and interactions between germline and soma are particularly important to safeguard germline proliferation and differentiation (Tran et al. 2000; Jemc 2011; Zoller and Schulz 2012). The mitotically active germ cells are prone to tumorigenesis. Other than the genes that suppress overproliferation autonomously in germ cells (Insco et al. 2009, 2012; Pek et al. 2009; Zhao et al. 2013; Chen et al. 2014), disruption of some genes, such as the components in bone morphogenetic protein (BMP) and epidermal growth factor receptor (EGFR) pathways, in somatic cells nonautonomously triggers germline excessive proliferation (Kiger et al. 2000; Tran et al. 

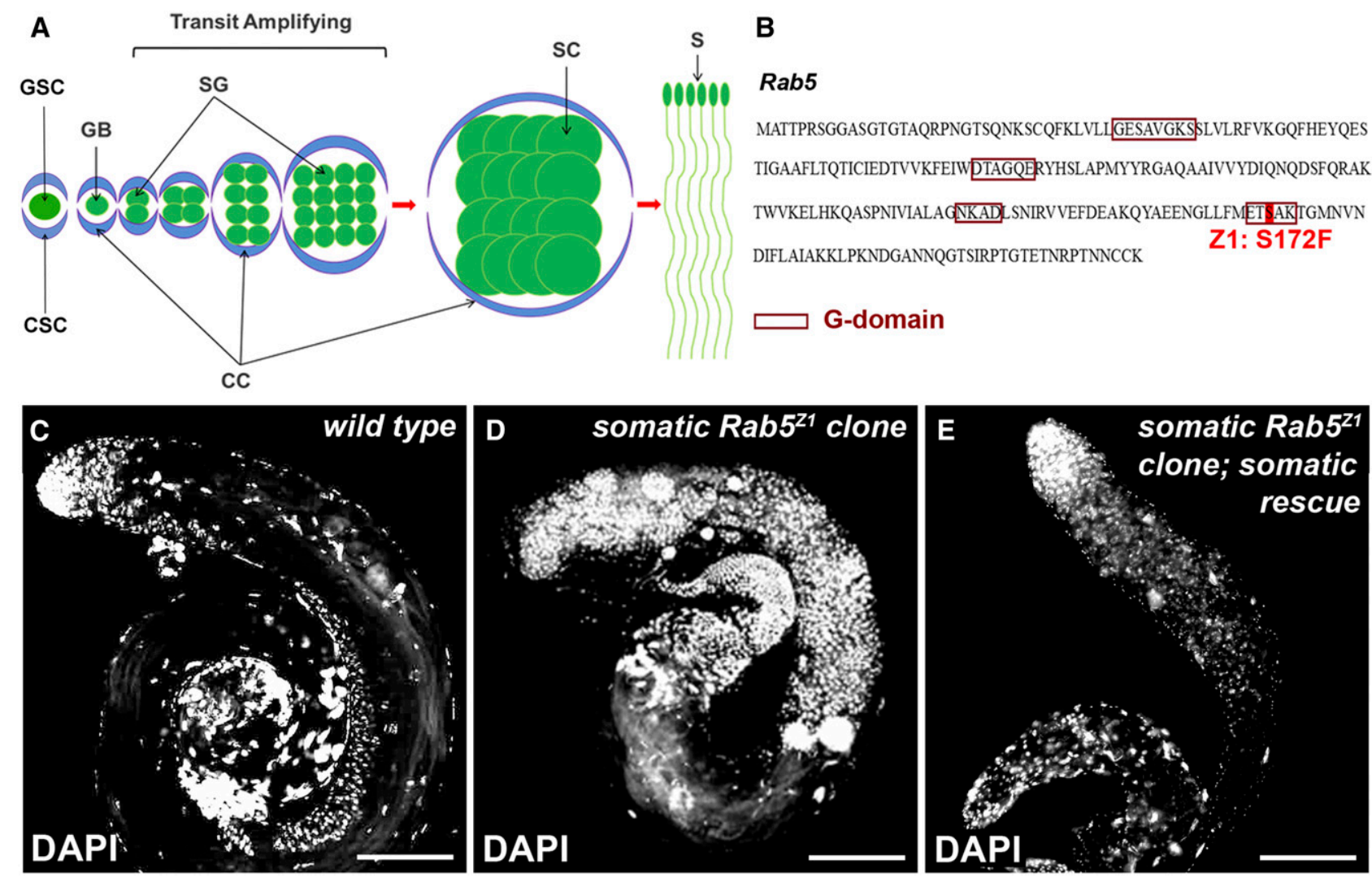

Figure 1 EMS mutagenesis generated a point mutation in the GTPase domain of Rab5. (A) Cartoon illustration of fly male germline cyst development. Germline in green, somatic cells in blue. GSC, germline stem cell; GB, gonialblast; SG, spermatogonium; SC, spermatocyte; S, sperm; CSC, cyst stem cell; CC, cyst cell. (B) Rab5 Z1 bears a S172F mutation in the GTPase domain of Rab5. Red boxes indicate the segments that form the GTPase domain. (C) A wild-type testis. The GSCs, GBs, and SGs at the apical part of the testis are brightly stained with DNA-dye, DAPI, due to their small size and chromosome packaging. The DAPI signal is less bright in SCs, which occupy the most space in the testis. (D) A testis containing somatic Rab5 $5^{21}$ clones induced by C587-GAL4 (genotype: C587-GAL4/Y; Rab521 FRT40A/GFP FRT40A; UAS-FLP/+). The early cells with intense DAPI signal dramatically increased. (E) Somatically introduced Rab5 rescued the phenotype caused by somatic Rab5 ${ }^{Z 1}$ clones (genotype: C587-GAL4/Y; Rab5 Z1 FRT40A/GFP FRT40A; UAS-FLP/ UAS-YFP-Rab5). Bar, $100 \mu \mathrm{m}$

2000; Schulz et al. 2002; Shivdasani and Ingham 2003; Li et al. 2007). But the signals generated in cyst cells and sensed by the germline are still elusive. These findings suggest that the control mechanism of fly germline proliferation can serve as an ideal system to investigate the role of nonautonomous effect on tumorigenesis.

Endocytosis is the main process by which cells maintain transmembrane protein homeostasis. The endocytic process involves internalization of the plasma membrane, early endosome entry, multivesicular body (MVB) sorting, and lysosome degradation (Kumari et al. 2010). Endocytic genes are considered tumor suppressor genes (TSG) (Vaccari and Bilder 2009), given that many signaling pathways are regulated by endocytosis. Disruption of main endocytic regulators in flies initiates a tumorigenic process that is reminiscent of human cases, displaying unrestrained proliferation, loss of cell polarity, and escape from apoptosis (Vaccari and Bilder 2009). Moreover, the mammalian homologs of certain endocytic TSGs act as tumor suppressors too, such as TSG101 (Li and Cohen 1996; Moberg et al. 2005) and HCRP1 (Xu et al. 2003; Bache et al. 2004), suggesting the conserved functions of endocytic TSGs.

We report here that in fly testis, the endocytic gene Rab5 functions in somatic cells to nonautonomously control germline mitotic divisions through inhibiting $d p p$ expression, and the JNK pathway mediates the regulation of $d p p$. We also found that multiple factors of endocytosis act in somatic cells to regulate germline amplification. These findings indicate that when the endocytic state of the surrounding cells is altered, genetically normal germ cells overgrow. This provides a simple model to dissect the relation between tumor and its niche.

\section{Materials and Methods}

\section{Fly genetics}

$R a b 5^{Z 1}$ was an EMS allele generated in our lab; $R a b 5^{2}$ was from Marcos González; UAS-Rab5 GFP/TM3, UAS-Rab5 $R N A i / C y O$, and UAS-Rab5 RNAi/TM3 were from Xinhua Lin; C587-GAL4 was from Ting Xie; nosGAL4VP16 was from Ruth Lehmann; puc ${ }^{E 69}$ (puc-lacZ) was from Joaquim Culi; kek1-lacZ is an enhancer trap line (BB142) from T. Schüpbach; 

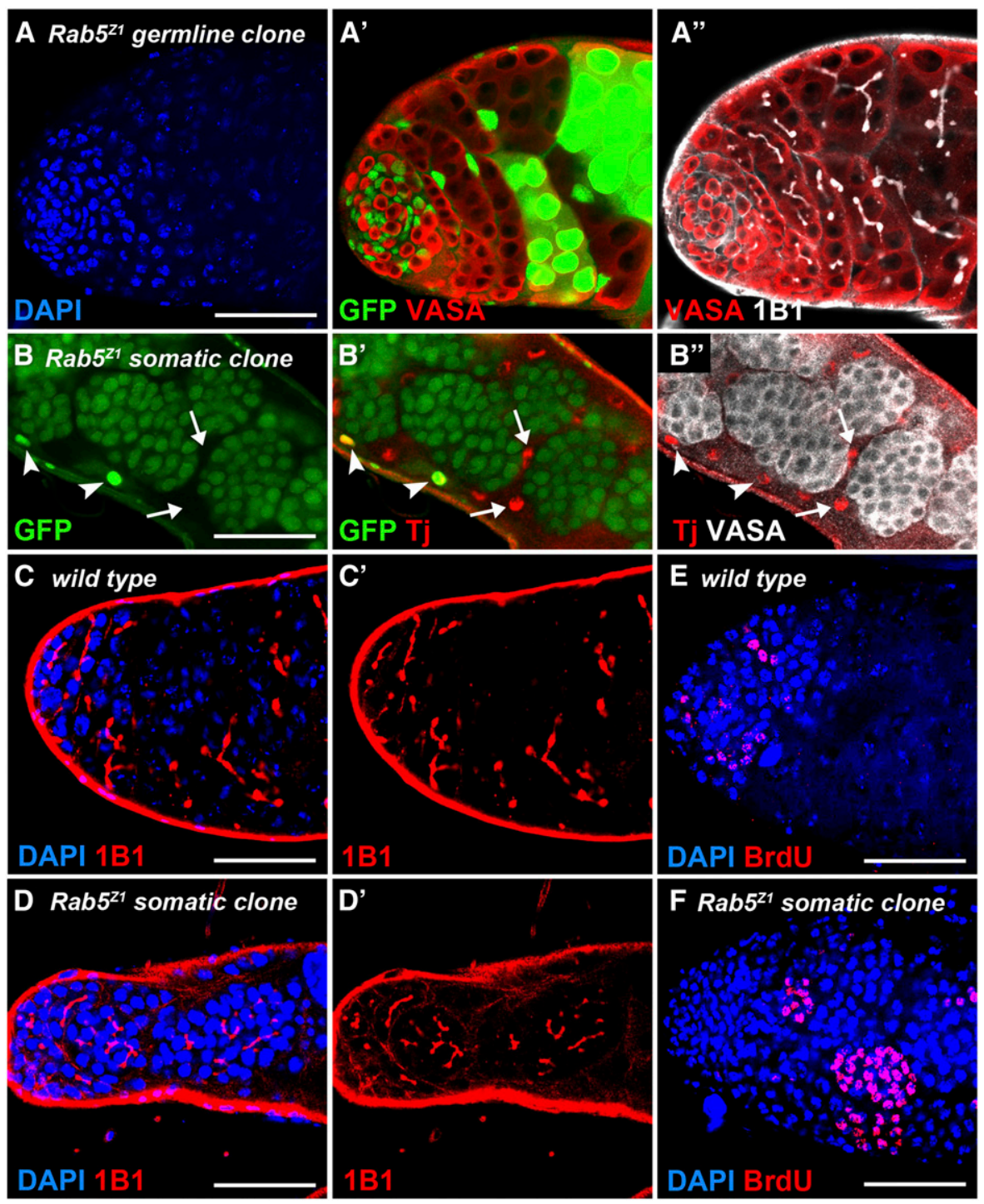

Figure 2 Rab5 acted in the somatic cells to restrict spermatogonial proliferation. (A and $B$ ) Rab5 in cyst cells is required to restrict germ cell proliferation. GFP-negative cells are Rab5 mutant and VASA is specific for germ cells. (A) A testis tip with germline Rab5 $5^{z 1}$ clones induced (genotype: Rab5 Z1 FRT4OA/GFP FRT40A; nos-GAL4/UAS-FLP). 1B1 immunostains the subcellular organelle, which is dot-shaped spectrosomes in GSCs and GBs, and branchshaped fusomes in SGs and SCs. (B) Somatic $R a b 5^{Z 1}$ clones were induced around midpupal stage by shifting to $29^{\circ}$ for 2 days (C587-GAL4; Rab5 ${ }^{21}$ FRT40A/GFP FRT40A; tub-GAL80/UAS-FLP). Arrowheads, Rab5+ cells; arrows, Rab5 $5^{71}$ mutant cells. Tj is a marker for cyst cells. (C-F) The overproliferating germ cells are spermatogonia. (C) A wild-type control for D. (D) Rab5 $5^{21}$ somatic clones led to overproliferation of SG, characterized by the branched fusomes. (E) BrdU labels the $S$ phase of cell cycle. GSCs and GBs divide independently and SGs in one cyst divide synchronously. (F) Clustered BrdU signal indicated the synchronous division of overproliferating germ cells. Genotype in $B, D$, and F: C587-GAL4/Y; Rab5Z1 FRT4OA/GFP FRT4OA; UAS-FLP/+. Bar, $50 \mu \mathrm{m}$.
UAS-YFP-Rab5 (BL24616), UAS-TSG101-RNAi (BL35710), UAS-Vps25-RNAi (BL26286), UAS-avl-RNAi (BL29546), UASCdc42-RNAi (BL28021), dpp ${ }^{P 10638}$ (dpp-lacZ), tj-GAL4, tub$G A L 80^{t s}$, UAS-dpp-RNAi (BL25782), UAS-bsk ${ }^{D N}$, UAS-hep ${ }^{C A}$, and $2 \mathrm{~L}$ deficiency flies were from Bloomington Drosophila Stock Center.

Fly stocks were maintained under standard culture conditions and all flies were dissected 0-2 days after eclosion unless otherwise indicated. For GAL4/GAL80 ${ }^{\text {ts }}$ controlled RNAi or gene expression, flies were raised at 18 or $25^{\circ}$, shifted to $29^{\circ}$ upon eclosion, and aged at $29^{\circ}$ for 5 days before dissection. Germline or somatic cyst cell specific clones were generated by expressing FLP with nosGAL4VP16 or C587GAL4 (Kawase et al. 2004), respectively.

\section{EMS mutagenesis}

Following standard protocol, isogenized flies bearing FRT and UAS-FLP were fed with EMS $(37.5 \mathrm{mM})$ overnight. The progenies of EMS-treated flies were crossed to those carrying FRT-GFP and nos-GAL4. Their male offspring were dissected and stained with DAPI. The original mutant line containing $R a b 5^{Z 1}$ was one of the mutants with germline overgrowth phenotype. However, once the mutant line was purified by recombination crosses, the phenotype was only induced by somatic mutant clones. Rab5 $5^{Z 1}$ was mapped by deficiency screens and candidate gene sequencing.

\section{BrdU labeling}

Testes were dissected in PBS, incubated for $1 \mathrm{hr}$ in PBS containing $100 \mu \mathrm{g} / \mathrm{ml} \mathrm{BrdU}$ (Sigma \#B5002) at $25^{\circ}$ and then fixed. The rest of the procedure was described previously (Wang and Mann 2003).

\section{Immunofluorescence}

Fly testes were prepared and immunostained as previously described (Wang and Mann 2003). The following antibodies were used: 1B1 (1:50, DSHB), rabbit anti-Bam (1:2000, a gift from Chen Dahua), mouse anti-BrdU (1:200, BD), mouse anti- $\beta$-Gal (40-1a 1:100, DSHB), rabbit anti-pSmad1/5 (1:200, Cell Signaling\#9516S), rabbit anti-STAT [1:4000; 
against (KLH)-GMADFDTITNFENF-OH], rabbit anti-Vasa [1:8000; against (KLH)-MSDDWDDEPIVDTRGARC-OH], guinea pig anti-Vasa (1:4000; against 6xHis-Vasa produced in Escherichia coli), rabbit anti-Zfh1 (1:4000; against 648-775 aa produced in $E$. coli, a gift from Zhao Yun), rabbit anti-Tj [1:1000; against (KLH)-CHHQWHMDERRLQPLSPP-OH and (KLH)-CAVEVSPSPHIKLRSFSG-OH].

\section{Permeability assay}

Testes were dissected in PBS, and transferred to PBS containing $10 \mathrm{kDa}$ lysine-fixable dextran conjugated to Cascade Blue (Invitrogen, Carlsbad, CA) at a final concentration of $0.5 \mu \mathrm{g} / \mu \mathrm{l}$, and incubated for $\sim 40 \mathrm{~min}$. The dye was washed off with $4 \%$ formaldehyde/PBS before subsequent fixation and immunostaining.

\section{Data availability}

The authors state that all data necessary for confirming the conclusions presented in the article are represented fully within the article. Strains are available upon request.

\section{Results}

\section{Rab5 is required in somatic cells to restrict germline proliferation}

In a fly testis, germline stem cells (GSCs) reside in the apex, and the differentiated cells of gonialblasts, spermatogonia, spermatocytes, and spermatids are orderly distributed toward the basal end (Figure 1, A and $\mathrm{C}$ ). The integrity and the activity of the GSCs contribute most to the tissue homeostasis. To avoid potential risk of mutations due to frequent cell divisions and meanwhile to provide plenty of progenies, germline stem cell lineage adopts a transit-amplifying (TA) strategy (Spradling et al. 2011). A gonialblast (the direct daughter cell of GSC) undergoes four rounds of mitotic divisions to amplify as spermatogonia before entering meiosis. The spermatogonia derived from a single gonialblast divide synchronously, interconnected with each other due to incomplete cytokinesis, and enveloped by two somatic cyst cells to form a developing unit, the cyst (Figure 1A). Failure to stop TA divisions results in spermatogonial (SG) tumor.

In a large-scale EMS screen searching for genes that restrict proliferation of germline stem cell lineage, we isolated a mutant line containing a point mutation in the GTPase domain of Rab5 (Figure 1B). We named this mutant allele $R a b 5^{Z 1}$, which was homozygous lethal and also lethal in trans with the null allele Rab52 (Wucherpfennig et al. 2003). To examine in which cells Rab5 is required, we performed mosaic analysis to generate either germline or somatic clones of Rab5 mutant cells in the testis. In wild-type testis, early stage cells (brightly stained by DAPI) only occupied a small region in the apical tip (Figure 1C). Inducing $R a b 5^{Z 1}$ clones in the somatic cells resulted in significant expansion of brightly DAPI-stained cells (Figure 1D). And this hyperplasia phenotype caused by $R a b 5^{Z 1}$ was blocked by restoring Rab5
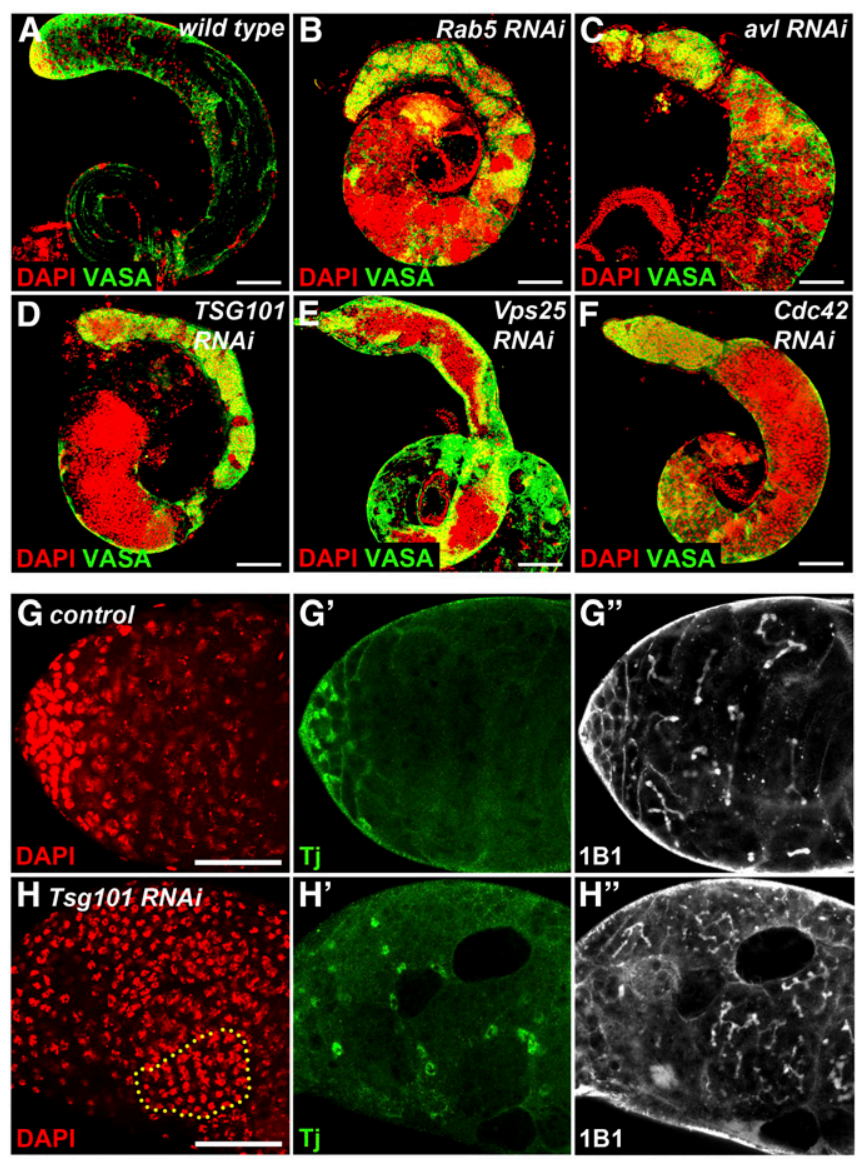

Figure 3 Knock-down of endocytic genes in somatic cells led to germline tumors. (A) A wild-type testis. (B) Knock-down of Rab5 in cyst cells (genotype: tj-GAL4/+; tub-GAL80ts/UAS-Rab5-RNAi) induced significant germline tumors. (C-E) Knock-down of avl (tj-GAL4/+; tub-GAL80ts/UASavl-RNAi), TSG101 (tj-GAL4/+; tub-GAL80ts/UAS-TSG101-RNAi), Vps25 (tj-GAL4/+; tub-GAL80ts/UAS-Vps25-RNAi), and Cdc42 (tj-GAL4/+; tub-GAL80ts/UAS-Cdc42-RNAi) in cyst cells generated germ cell overproliferation phenotype similar to that of Rab5. (G-G") The control for TSG101 RNAi (tub-GAL80ts/UAS-TSG101-RNAi). (H-H") TSG101 RNAi (tj-GAL4/+; tub-GAL80ts/UAS-TSG101-RNAi), a SG tumor is circled. Bars, $100 \mu \mathrm{m}(\mathrm{A}-\mathrm{F}) ; 50 \mu \mathrm{m}$ ( $\mathrm{G}$ and $\mathrm{H})$

in the somatic cells (Figure 1E). Interestingly, when $R a b 5^{Z 1}$ mutant clones were generated in the germ cells, the germline developed normally (Figure 2A). Whereas, somatic Rab5 $5^{Z 1}$ mutant clones caused early stage germ cells to overproliferate (Figure 2B). This nonautonomous effect of Rab5 was further confirmed by RNA interference (RNAi) assay of Rab5 in somatic cells (Figure 3B).

Did Rab5 mutant cyst cells become CSC (cyst stem cell)like cells to simulate germ cell overgrowth? We checked the cell fate of Rab5 mutant somatic cells. Zfh1 is a transcription factor highly expressed in CSCs, whereas $\mathrm{Tj}$ is present in both CSCs and differentiated cyst cells (Supplemental Material, Figure S1, A-C and Figure S2E in File S1). In the early somatic mutant clones of Rab5, we did not detect high levels of Zfh1 but only Tj (Figure S1, A-C in File S1). In the cyst cells around the tumor, Zfh1-positive cells were occasionally detected (Figure S1, D-F in File S1), and not in tight association 


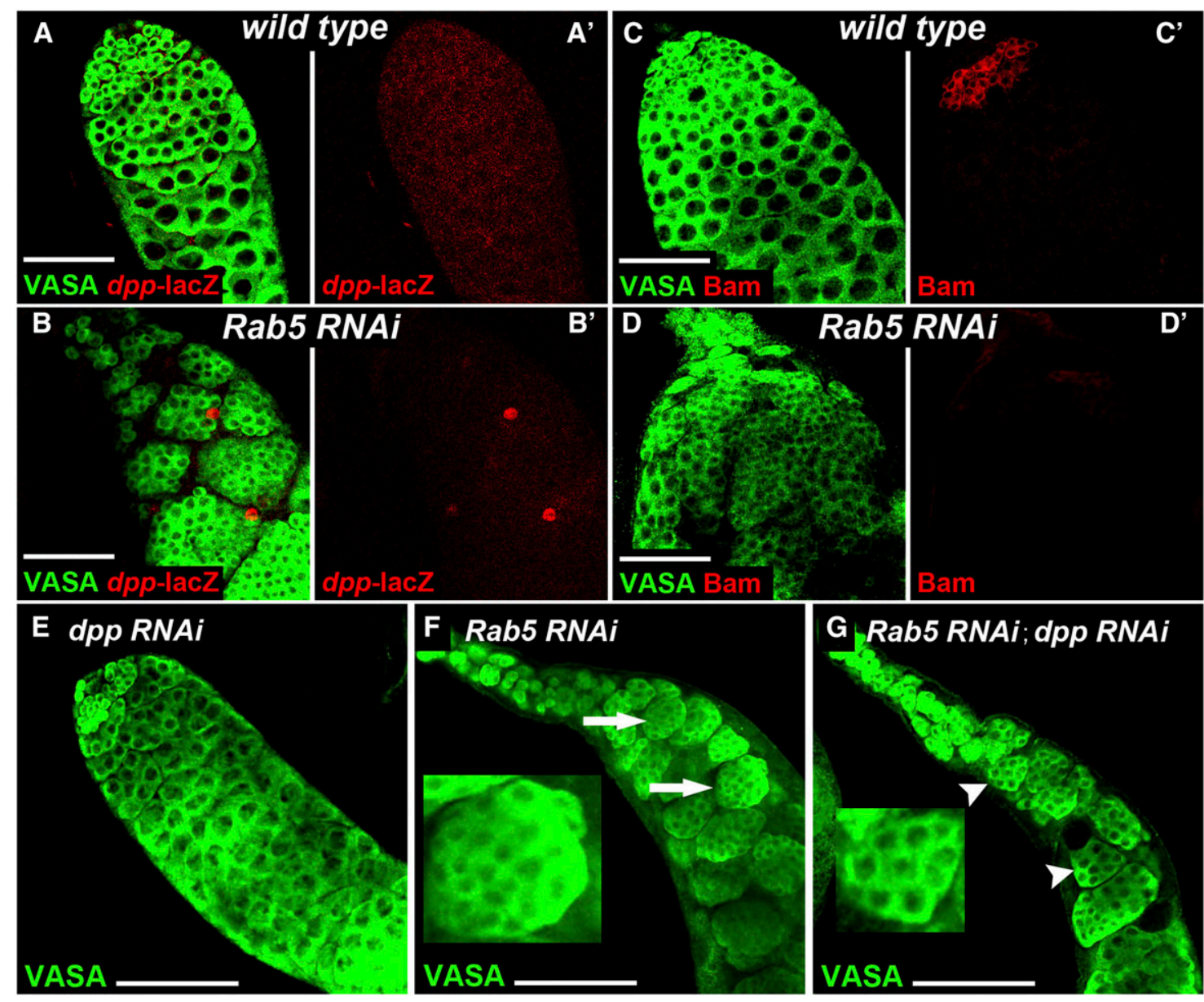

H

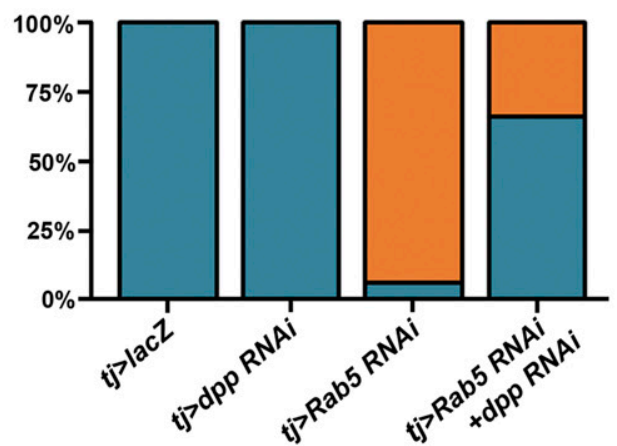

no over-proliferation

over-proliferation

Figure 4 Rab5 prohibited germline overamplification by transcriptionally repressing $d p p$. (A and B) Rab5 represses $d p p$ expression transcriptionally. $d p p$-lacZ is the expression reporter of $d p p$, which was not detectable in wild-type testis (A), but was detected in cyst cells when Rab5 is knocked down in cyst cells (B, genotype: tj-GAL4 dpp-lacZIUAS-Rab5-RNAi; tubGAL80ts/+). (C and D) Rab5 promotes Bam expression. Bam is normally expressed in spermatogonia (C), but barely detected in tumorigenic spermatogonia in Rab5 RNAi testis (D, genotype: tj-GAL4/UAS-Rab5-RNAi; tub-GAL80ts/+). (E-H) Somatic RNAi of dpp blocks the germline overproliferation induced by somatic Rab5 RNAi. Solely knocking down dpp in cyst cells exhibits no phenotype (E, genotype: tj-GAL4/+; tubGAL80ts/UAS-dpp-RNAi). (F) Testis with somatic Rab5 RNAi (genotype: tj-GAL4/UASRab5-RNAi; tub-GAL80ts/+) is full of overproliferating cysts that contain far more than 16 germ cells (arrows indicate higher magnification shown in inset). (G) Simultaneous knock-down of dpp and Rab5 (genotype: tj-GAL4/UAS-Rab5-RNAi; tubGAL80ts/UAS-dpp-RNAi) restored the germ cell number to 16 per cyst, as in normal situation (arrowheads indicate higher magnification shown in inset). (H) Percentage of testes containing SG tumors. $t j>$ lacZ (genotype: tj-GAL4/+; tub-GAL80 ${ }^{t s} /$ UAS-lacZ) serves as a parallel control. The number of testes scored for each genotype from left to right: $n=42,38,35$, and 35, respectively. A testis of "overproliferation" was determined by the presence of at least one cyst containing $>16$ spermatogonia in a testis. Bars, $50 \mu \mathrm{m}(A-D) ; 100 \mu \mathrm{m}(E-G)$. 


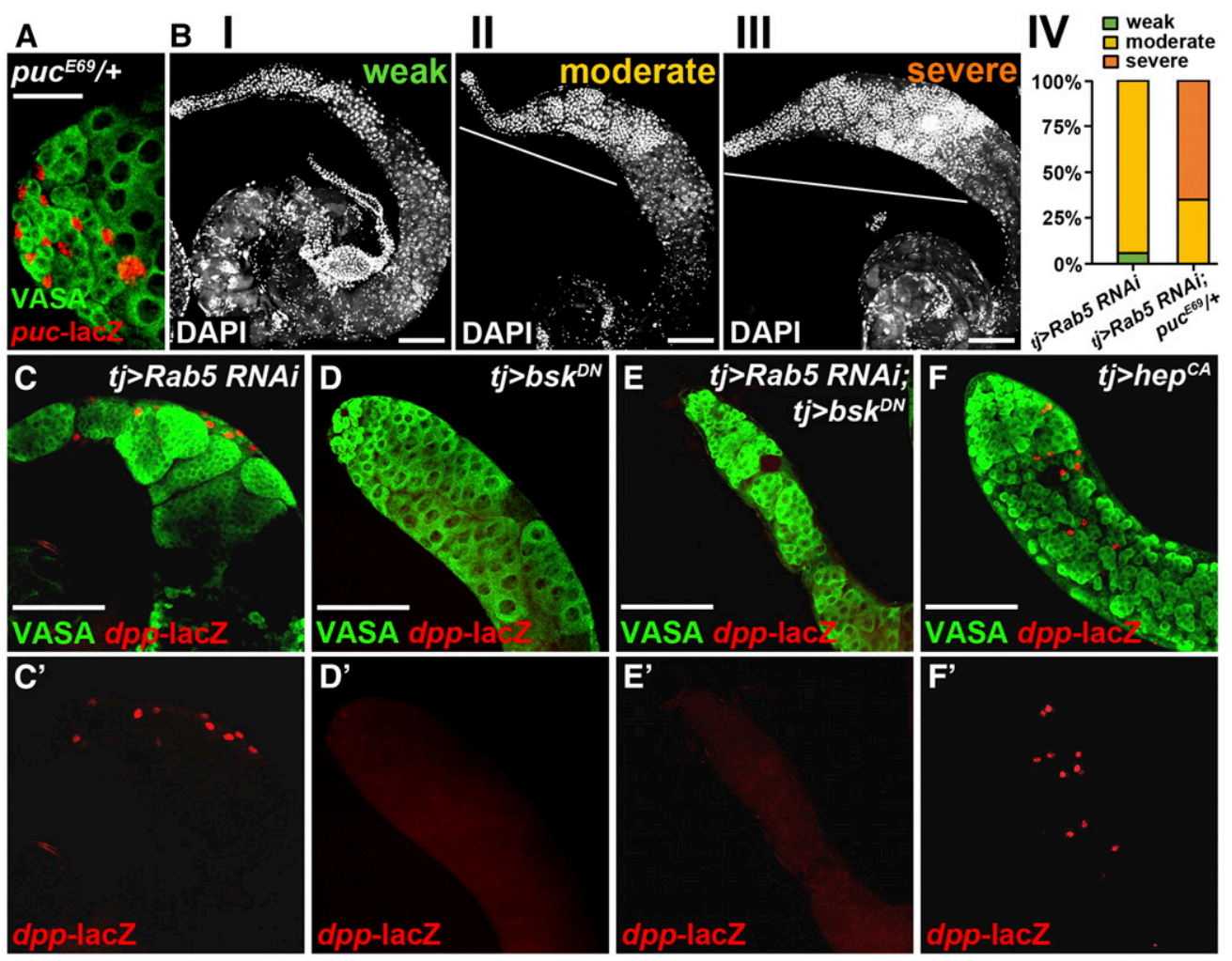

Figure 5 JNK pathway mediated Rab5's negative regulation on $d p p$. (A) puc is expressed in cyst cells. puc-lacZ is a P-element insertion in the gene puc, which generated a loss-of-function allele, puc ${ }^{E 69}$, meanwhile serving as a transcriptional reporter of puc. Only the apical tip of a testis is shown. (B) Removal of one copy of puc enhanced the tumorigenic phenotype of Rab5 RNAi (genotype: tj-GAL4/UAS-Rab5-RNAi; tubGAL80ts/puc $\left.{ }^{E 69}\right)$. The testes containing SG tumor are divided into weak (SG accumulation $<1 / 3$ of testis mass), moderate (SG accumulation $\sim 1 / 3-1 / 2$ of testis mass), and severe (SG accumulation $\geq 1 / 2$ of testis mass) categories. Examples are given in BI-BIII. The white lines indicate the segments occupied by tumor cells. The scoring of each category in different genotypes is presented in BIV. The numbers of testes scored in BIV are: $t j>$ Rab5 RNAi, $n=35$; tj > Rab5 RNAi; puc E69/+, $n=43$. (C-E) Expression of bsk ${ }^{D N}$ in somatic cells suppressed the $d p p$ expression and germline overproliferation phenotype of somatic Rab5 RNAi. (C) Genotype: tj-GAL4 dpp-lacZIUAS-Rab5-RNAi; tubGAL80ts/+. (D) Genotype: UAS-bskDN/Y; tj-GAL4 dpp-lacZ/+; tubGAL80ts/+. (E) Genotype: UAS-bsk $D N / Y$; tj-GAL4 dpp-lacZIUAS-Rab5-RNAi; tubGAL80ts/t. (F) Somatic expression of hep CA induced ectopic dpp expression in cyst cells and led to SG accumulation (genotype: tj-GAL4 dpp-lacZ/+; tubGAL80ts/UAS-hep CA). Bars, $25 \mu \mathrm{m}(\mathrm{A}) ; 100 \mu \mathrm{m}$ (B-F).

with Rab5 mutant clones (Figure S1, D and E in File S1). These results indicate that compromising Rab5 did not turn the cyst cells into CSC-like cells.

Since the mitotically active germ cells at the apical end of the testis consist of GSCs, gonialblast and spermatogonia, all of which are similar in cell size and morphology, we examined the specific stage of the overproliferating germ cells. Branched fusomes, indicating incomplete cytokinesis, and clustered BrdU labeling, a sign of synchronous division, were observed in the germline tumors (Figure 2, C-F). These two characters decently distinguish spermatogonia from other mitotic germ cells. Thus, we conclude that Rab5 acts in somatic cells to restrict spermatogonial mitotic amplification.

\section{Endocytic genes are required in somatic cells to regulate germ cell development}

Rab5 is a conserved small GTPase, which functions as a key factor of early endocytosis. Activated Rab5 is important for sequestering ligands into clathrin-coated pits and the subsequent fusion of vesicles with early endosomes (Wucherpfennig et al. 2003). To determine whether or not somatic Rab5 mutation-induced germline overamplification is due to impaired endocytosis in the surrounding somatic cyst cells, we checked the function of multiple endocytosisrelated genes in this process. avl (syntaxin7) functions in early endosome entry as Rab5 (Lu and Bilder 2005), while TSG101 and Vps25 are both essential for MVB sorting
(Moberg et al. 2005; Thompson et al. 2005). Cdc42 promotes clathrin-independent endocytic process (Chen et al. 2016). Knock-down of avl, TSG101, Vps25, or Cdc42 in the cyst cells also led to dramatic germline hyperplasia, the same as the knock-down of Rab5 (Figure 3, A-F). The presence of cyst cells around SG tumor indicates that compromising TSG101 did not block cyst cell division (Figure $3, \mathrm{G}$ and $\mathrm{H}, \mathrm{Tj}$ is a cyst cell marker). Interestingly, knocking down any of these endocytic genes in germ cells did not lead to germline amplification defect (data not shown). This suggests that the integrity of endocytic process in the somatic cells is required to maintain normal germ cell development.

\section{Rab5 represses dpp transcription to prevent germline overamplification}

Endocytosis is reported in other tissues to have a critical role in the transduction of signaling pathways, such as EGFR, Notch, WNT, and BMP (Vaccari and Bilder 2009). In Drosophila spermatogenesis, EGFR signaling in somatic cyst cells is known to constrain germ cells from overamplification, with its ligand secreted from germ cells and received by cyst cells (Schulz et al. 2002; Hudson et al. 2013). The disruption of multiple components of BMP signaling in the cyst cells also leads to germline tumor (Kawase et al. 2004; Li et al. 2007; Yu et al. 2016), although the specific mechanism is not clarified. Is EGFR or BMP signaling altered and responsible for germ cell overproliferation when Rab5 is compromised? 


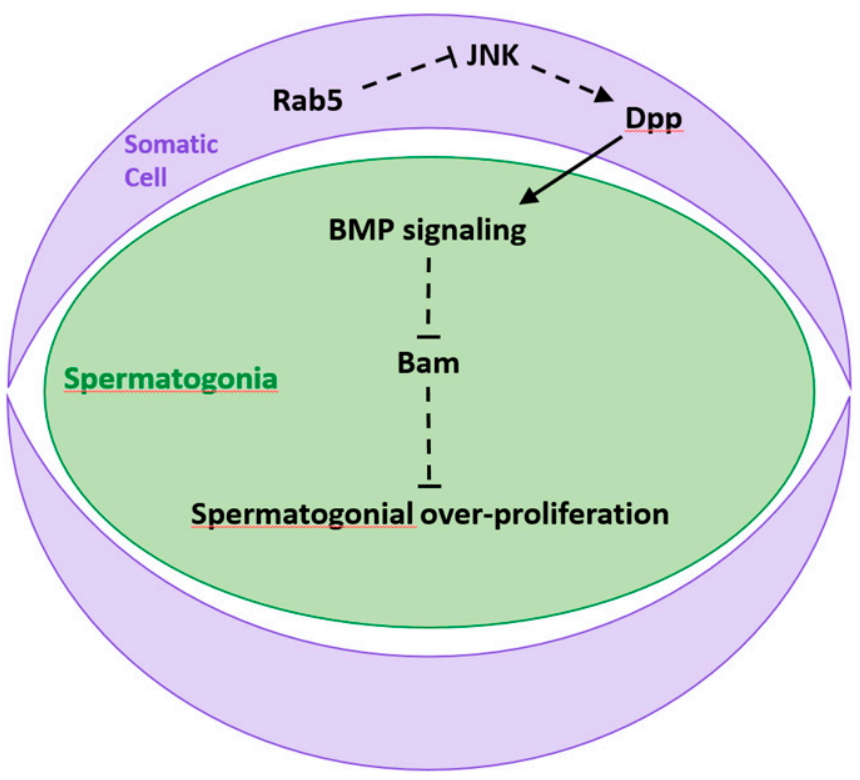

Figure 6 A model of how Rab5 controls spermatogonial amplification from somatic cells. Pointed arrows indicate promoting effect. Blunt arrows indicate repressive effect. Dotted lines infer indirect or potential effect. Dpp is not necessarily generated from the somatic cell encysting the spermatogonia.

kek1-lacZ is a downstream reporter of EGFR signaling and is normally expressed in cyst cells (Figure S2, C and E in File $\mathrm{S} 1$ ), reflecting this pathway's role in spermatogenesis. When Rab5 was knocked down in cyst cells, kek1-lacZ expression around the overproliferating germ cells was not obviously changed (Figure S2, D and F in File S1), suggesting that EGFR signaling was not substantially affected.

dad-lacZ is an enhancer trap insertion and serves as the BMP signaling reporter. When Rab5 ${ }^{Z 1}$ somatic clones were generated, dad-lacZ detected in the Rab5 mutant cyst cell clones showed no obvious difference from that in the wildtype cyst cells (Figure S2G in File S1), suggesting BMP signaling was not decreased by the loss of Rab5.

Although Rab5 was not involved in EGFR or BMP signaling transduction in somatic cyst cells, we found that when Rab5 was knocked down in somatic cells, $d p p$ (fly homolog of BMP) was ectopically expressed in cyst cells. We determined $d p p$ expression by a lacZ enhancer trap, which faithfully reflects its endogenous expression pattern at the anterior/posterior boundary of imaginal discs. In a wild-type testis, dpp-lacZ could not be detected, not even in the hub region (Figure 4A and Figure S3, A' and C' in File S1). When Rab5 was knocked down, however, $d p p$-lacZ was detected in somatic cells (Figure 4B and Figure S3, B' and D' in File S1), indicating an ectopic increase of $d p p$ at the transcription level. We further tested whether this upregulation of $d p p$ was required for germline overproliferation. Indeed, knock-down of $d p p$ suppressed the germ cell overproliferation caused by Rab5 RNAi to a great extent (Figure 4, E-H).

It is reported that BMP signaling suppresses bam to prevent GSCs' premature differentiation (Chen and McKearin 2003;
Fuller and Spradling 2007). In male germline, accumulation of Bam is the key to control SG proliferation vs. differentiation. A low level of Bam promotes proliferation, while its high level initiates differentiation (Fuller and Spradling 2007; Insco et al. 2009). Normally Bam is accumulated in the 2to 8-cell stage of spermatogonia in the absence of Dpp (Figure 4C). We found that in the tumorigenic germ cells induced by Rab5 RNAi, Bam immunostaining was apparently decreased (Figure 4D), consistent with the elevated Dpp expression in the surrounding cyst cells. This suggests that Rab5-mutant somatic cells activated the BMP pathway in germ cells, though pMad (phosphorylated Smad1 homolog) was not detected in the SG tumor (Figure S3, A and B in File S1).

\section{JNK pathway mediates Rab5's negative regulation of dpp}

Since Rab5 is not likely to repress $d p p$ transcription directly, what signaling pathway mediates this repression? After screening several pathways that are involved in the mitotic division or cell growth (data not shown), we noticed that sustained JNK signaling activation in somatic cells also caused germ cell overproliferation. It has previously been found that the JNK signaling pathway is abnormally activated in cells with impaired endocytosis (Woodfield et al. 2013; Takino et al. 2014).

We further analyzed the genetic relationship of JNK signaling and Rab5 in terms of dpp expression and germ cell proliferation. puc, which is expressed in the early cyst cells in testis (Figure 5A), is the downstream target and an inhibitor of the JNK pathway (Biteau et al. 2011). Removing one copy of puc enhanced the tumorigenic phenotype triggered by reducing Rab5 (Figure 5B). bsk is the JNK kinase in Drosophila, and hep is the JNKK kinase upstream of bsk (Biteau et al. 2011). In the wild-type background, block of the JNK pathway by introducing the dominant negative form of $b s k, b s k^{D N}$, in somatic cells caused no phenotype (Figure 5D), and a similar observation was also made with bsk RNAi (data not shown). In the Rab5 knock-down background, the presence of $b s k^{D N}$ repressed ectopic expression of $d p p$ and significantly prevented the germ cells from overproliferation (Figure 5, C-E). In addition, the persistent expression of the constitutively active form of hep, hep ${ }^{C A}$, in the somatic cells was sufficient to induce ectopic $d p p$ signal and elicit germ cell overamplification (Figure 5F), suggesting the critical role of the JNK pathway in promoting $d p p$ expression. Therefore, the JNK pathway mediates the transcriptional suppression of $d p p$ by Rab5.

\section{Discussion}

In Drosophila testis, somatic cyst cells function as a niche to maintain the development of the germline lineage. The disruption of the cyst cell integrity nonautonomously leads to abnormal germline development in many previous reports (Kiger et al. 2000; Tran et al. 2000), and here in our case, tumorigenesis. This is reminiscent of the cancer stem cell 
niche in mammals. Although non-cell-autonomous effects of the niche on tumor cells are frequently reported, the molecular mechanisms remain to be elucidated.

Based on our data presented in this study, we propose that Rab5 negatively regulates the JNK signaling pathway to prohibit $d p p$ expression in the somatic cells in spermatogenesis. In the absence of the ligand Dpp, the prodifferentiation factor Bam, which is negatively regulated by BMP signaling, is expressed and accumulated to restrict mitotic amplification of germ cells (Figure 6). When Rab5 is downregulated in cyst cells, underdifferentiated germline cells overproliferate due to the nonautonomous effect of the BMP ligand Dpp.

\section{What mediated the BMP signaling in these overgrowing germ cells?}

BMP signaling represses Bam expression in the GSCs to maintain the stem cell fate, with its ligand secreted from CSCs (Chen and McKearin 2003; Kawase et al. 2004). In the spermatogonia, however, Bam is derepressed to promote differentiation, implying the requisite of inactivation of BMP signaling. In Rab5 knocked down testis, $d p p$-lacZ signal was always present around the SG tumors (Figure 4B, Figure 5C, and Figure S3, B and D in File S1), though not in direct contact with the tumors. Interestingly, the overamplifying cysts showed higher permeability, which might facilitate the Dpp signal to reach the germ cells inside the cysts (Figure S2, $A$ and $B$ in File S1).

Although the BMP signaling reporter dad-lacZ or pMad was not detectable in the overproliferating germ cells caused by compromised Rab5 in somatic cells, the requirement of Dpp expression in somatic cells and the decreased Bam in SG tumor is consistent with the activation of BMP pathways in germ cells. It is possible that under this circumstance, the BMP signaling pathway may activate effectors other than pMad, and Dad, especially Smox (dSmad2), has been found to mediate BMP signaling in testis (Li et al. 2007).

\section{Rab5 may be involved in multiple signaling pathways}

Disruption of Rab5 in the somatic cells induced an ectopic expression of $d p p$, which is mediated by the JNK signaling pathway. We noticed that in the Rab5 RNAi situation, although decreasing dpp level by downregulating JNK signaling could efficiently reduce the germ cell number per cyst back to 16, many cysts did not resume further differentiation. It is probably due to the fact that the JNK pathway is negatively regulated by Rab5 to inhibit $d p p$ expression and restrict excessive germ cell division, but not to promote differentiation. It is also noticeable that, although EGFR signaling reporter kek1-lacZ was not significantly downregulated around the tumor (Figure S2, C and D in File S1), the overproliferating germ cells exhibited abnormal encystment and elevated permeability (Figure S2, A and B in File S1), which could be due to a subtle disruption of the EGFR pathway. Thus, some signaling pathways required for differentiation may also be disrupted when Rab5 is compromised, indicating that multiple pathways are regulated by Rab5-involved endocytosis.

\section{Model of nonautonomous tumorigenesis}

Our discovery of somatic Rab5's control on germ cell proliferation provides a nonautonomous tumorigenesis model, where genetically mutant somatic cyst cells stimulate genetically normal germ cells to overgrow. Although it is previously reported that in fly epithelial tissue, loss of Rab5 nonautonomously caused tumor growth through the upregulation of Upd (IL-6 homolog) (Takino et al. 2014), here we report a different scenario between two distinct cell types in fly testis. In the former studies, Rab5 mutant clones in conjunction with activated oncogenic factor or cell death inhibitor induced the overgrowth of surrounding tissues (Takino et al. 2014). In our findings, the disruption of Rab5 alone was sufficient to induce nonautonomous overproliferation via BMP/Dpp release.

Microenvironment is known to contribute greatly in tumorigenesis. An exemplary illustration is that in human breast cancer, where active stromal fibroblasts are responsible for tumor growth, expansion, and metastasis in a non-cellautonomous manner via paracrine procarcinogenic effects (Al-Ansari and Aboussekhra 2015). Recently, some mediators and pathways responsible for this nonautonomous effect have been identified (Al-Rakan et al. 2016). In our model, the somatic cyst cells control germ cell proliferation also through paracrine signal transduction. In addition, we demonstrated that the genetically normal cells are capable of tumorigenesis, merely induced by the extrinsic signals such as BMP, and the tumors could be suppressed by just altering a gene expression from the surrounding cells. This provides a simple model to study how tumor develops in genetically altered niche cells and to search for potential therapeutic strategies.

\section{Acknowledgments}

We are deeply grateful to Marcos González, Dahua Chen, Xinhua Lin, Ting Xie, Ruth Lehmann, and Joaquim Culi for sharing research materials; to Developmental Studies of Hybridoma Bank and Bloomington Drosophila Stock Center for providing research materials. This work was supported by National Key Basic Research Program of China (2013CB945003) and National Science Foundation China (31471345). The authors declare no conflict of interest.

\section{Literature Cited}

Al-Ansari, M. M., and A. Aboussekhra, 2015 miR-146b-5p mediates p16-dependent repression of IL- 6 and suppresses paracrine procarcinogenic effects of breast stromal fibroblasts. Oncotarget 6: 30006-30016.

Al-Rakan, M. A., S. F. Hendrayani, and A. Aboussekhra, 2016 CHEK2 represses breast stromal fibroblasts and their paracrine tumor-promoting effects through suppressing SDF-1 and IL-6. BMC Cancer 16: 575.

Bache, K. G., T. Slagsvold, A. Cabezas, K. R. Rosendal, C. Raiborg et al., 2004 The growth-regulatory protein HCRP1/hVps37A is a subunit of mammalian ESCRT-I and mediates receptor downregulation. Mol. Biol. Cell 15: 4337-4346. 
Banfi, S., G. Borsani, E. Rossi, L. Bernard, A. Guffanti et al., 1996 Identification and mapping of human cDNAs homologous to Drosophila mutant genes through EST database searching. Nat. Genet. 13: 167-174.

Biteau, B., J. Karpac, D. Hwangbo, and H. Jasper, 2011 Regulation of Drosophila lifespan by JNK signaling. Exp. Gerontol. 46: 349-354.

Chen, D., and D. McKearin, 2003 Dpp signaling silences bam transcription directly to establish asymmetric divisions of germline stem cells. Curr. Biol. 13: 1786-1791.

Chen, D., C. Wu, S. Zhao, Q. Geng, Y. Gao et al., 2014 Three RNA binding proteins form a complex to promote differentiation of germline stem cell lineage in Drosophila. PLoS Genet. 10: e1004797.

Chen, H., Z. Gao, C. He, R. Xiang, T. H. van Kuppevelt et al., 2016 GRP75 upregulates clathrin-independent endocytosis through actin cytoskeleton reorganization mediated by the concurrent activation of Cdc42 and RhoA. Exp. Cell Res. 343: 223236.

Fuller, M. T., and A. C. Spradling, 2007 Male and female Drosophila germline stem cells: two versions of immortality. Science 316: 402-404.

Hudson, A. G., B. B. Parrott, Y. Qian, and C. Schulz, 2013 A temporal signature of epidermal growth factor signaling regulates the differentiation of germline cells in testes of Drosophila melanogaster. PLoS One 8: e70678.

Insco, M. L., A. Leon, C. H. Tam, D. M. McKearin, and M. T. Fuller, 2009 Accumulation of a differentiation regulator specifies transit amplifying division number in an adult stem cell lineage. Proc. Natl. Acad. Sci. USA 106: 22311-22316.

Insco, M. L., A. S. Bailey, J. Kim, G. H. Olivares, O. L. Wapinski et al., 2012 A self-limiting switch based on translational control regulates the transition from proliferation to differentiation in an adult stem cell lineage. Cell Stem Cell 11: 689-700.

Ito, S., T. Ueda, A. Ueno, H. Nakagawa, H. Taniguchi et al., 2014 A genetic screen in Drosophila for regulators of human prostate cancer progression. Biochem. Biophys. Res. Commun. 451: 548-555.

Jemc, J. C., 2011 Somatic gonadal cells: the supporting cast for the germline. Genesis 49: 753-775.

Kawase, E., M. D. Wong, B. C. Ding, and T. Xie, 2004 Gbb/Bmp signaling is essential for maintaining germline stem cells and for repressing bam transcription in the Drosophila testis. Development 131: 1365-1375.

Kiger, A. A., H. White-Cooper, and M. T. Fuller, 2000 Somatic support cells restrict germline stem cell self-renewal and promote differentiation. Nature 407: 750-754.

Kumari, S., S. Mg, and S. Mayor, 2010 Endocytosis unplugged: multiple ways to enter the cell. Cell Res. 20: 256-275.

Li, C. Y., Z. Guo, and Z. Wang, 2007 TGFbeta receptor saxophone non-autonomously regulates germline proliferation in a Smox/ dSmad2-dependent manner in Drosophila testis. Dev. Biol. 309: 70-77.

Li, L., and S. N. Cohen, 1996 Tsg101: a novel tumor susceptibility gene isolated by controlled homozygous functional knockout of allelic loci in mammalian cells. Cell 85: 319-329.

Lu, H., and D. Bilder, 2005 Endocytic control of epithelial polarity and proliferation in Drosophila. Nat. Cell Biol. 7: 1232-1239.

Moberg, K. H., S. Schelble, S. K. Burdick, and I. K. Hariharan, 2005 Mutations in erupted, the Drosophila ortholog of mammalian tumor susceptibility gene 101 , elicit non-cell-autonomous overgrowth. Dev. Cell 9: 699-710.

Nemeth, Z., E. Csizmadia, L. Vikstrom, M. Li, K. Bisht et al., 2016 Alterations of tumor microenvironment by carbon monoxide impedes lung cancer growth. Oncotarget 7: 2391923932.
Pek, J. W., A. K. Lim, and T. Kai, 2009 Drosophila maelstrom ensures proper germline stem cell lineage differentiation by repressing microRNA-7. Dev. Cell 17: 417-424.

Reilly, K. M., and T. Van Dyke, 2008 It takes a (dysfunctional) village to raise a tumor. Cell 135: 408-410.

Rubin, G. M., M. D. Yandell, J. R. Wortman, G. L. Gabor Miklos, C. R. Nelson et al., 2000 Comparative genomics of the eukaryotes. Science 287: 2204-2215.

Schulz, C., C. G. Wood, D. L. Jones, S. I. Tazuke, and M. T. Fuller, 2002 Signaling from germ cells mediated by the rhomboid homolog stet organizes encapsulation by somatic support cells. Development 129: 4523-4534.

Shivdasani, A. A., and P. W. Ingham, 2003 Regulation of stem cell maintenance and transit amplifying cell proliferation by tgf-beta signaling in Drosophila spermatogenesis. Curr. Biol. 13: 20652072.

Spradling, A., M. T. Fuller, R. E. Braun, and S. Yoshida, 2011 Germline stem cells. Cold Spring Harb. Perspect. Biol. 3: a002642.

Tabassum, D. P., and K. Polyak, 2015 Tumorigenesis: it takes a village. Nat. Rev. Cancer 15: 473-483.

Takino, K., S. Ohsawa, and T. Igaki, 2014 Loss of Rab5 drives non-autonomous cell proliferation through TNF and Ras signaling in Drosophila. Dev. Biol. 395: 19-28.

Thompson, B. J., J. Mathieu, H. H. Sung, E. Loeser, P. Rorth et al., 2005 Tumor suppressor properties of the ESCRT-II complex component Vps25 in Drosophila. Dev. Cell 9: 711-720.

Tissot, T., B. Ujvari, E. Solary, P. Lassus, B. Roche et al., 2016 Do cell-autonomous and non-cell-autonomous effects drive the structure of tumor ecosystems? Biochim. Biophys. Acta 1865: 147-154.

Tran, J., T. J. Brenner, and S. DiNardo, 2000 Somatic control over the germline stem cell lineage during Drosophila spermatogenesis. Nature 407: 754-757.

Vaccari, T., and D. Bilder, 2009 At the crossroads of polarity, proliferation and apoptosis: the use of Drosophila to unravel the multifaceted role of endocytosis in tumor suppression. Mol. Oncol. 3: 354-365.

Wang, Z., and R. S. Mann, 2003 Requirement for two nearly identical TGIF-related homeobox genes in Drosophila spermatogenesis. Development 130: 2853-2865.

Woodfield, S. E., H. K. Graves, J. A. Hernandez, and A. Bergmann, 2013 De-regulation of JNK and JAK/STAT signaling in ESCRT-II mutant tissues cooperatively contributes to neoplastic tumorigenesis. PLoS One 8: e56021.

Wucherpfennig, T., M. Wilsch-Brauninger, and M. Gonzalez-Gaitan, 2003 Role of Drosophila Rab5 during endosomal trafficking at the synapse and evoked neurotransmitter release. J. Cell Biol. 161: 609-624.

Xu, Z., L. Liang, H. Wang, T. Li, and M. Zhao, 2003 HCRP1, a novel gene that is downregulated in hepatocellular carcinoma, encodes a growth-inhibitory protein. Biochem. Biophys. Res. Commun. 311: 1057-1066.

Yu, J., Y. Liu, X. Lan, H. Wu, Y. Wen et al., 2016 CHES-1-like, the ortholog of a non-obstructive azoospermia-associated gene, blocks germline stem cell differentiation by upregulating Dpp expression in Drosophila testis. Oncotarget 7: 42303-42313.

Zhao, S., D. Chen, Q. Geng, and Z. Wang, 2013 The highly conserved LAMMER/CLK2 protein kinases prevent germ cell overproliferation in Drosophila. Dev. Biol. 376: 163-170.

Zoller, R., and C. Schulz, 2012 The Drosophila cyst stem cell lineage: partners behind the scenes? Spermatogenesis 2: 145-157.

Communicating editor: M. F. Wolfner 